\title{
CONSTRUÇÃO ANALÍTICA DE SEMIVARIOGRAMAS MÉDIOS LINEARES PARA KRIGAGEM DE BLOCOS
}

\author{
Ícaro Viterbre Debique Sousa1, Heron Viterbre Debique Sousa², Antonio Mendes Magalhães \\ Júnior $^{3}$, Pedro Henrique Nunes ${ }^{4}$, Iuri dos Santos Manoel $^{5}$, Helena Gonçalves Ikeda ${ }^{6}$, Rogério \\ Paes Menezes Filho ${ }^{7}$, Aliff Junio de Castro Faria ${ }^{8}$, Alessandro Leonardo da Silva ${ }^{9}$, Marcelo \\ Robert Fonseca Gontijo $^{10}$, Rodrigo Silva Fonseca ${ }^{11}$ and Thais Prado Vasconcelos Silva ${ }^{12}$
}

${ }^{1}$ Doutorando em Estatística e Experimentação Agropecuária, Departamento de Estatística, Universidade Federal de Lavras;

${ }^{2}$ Graduando em Engenharia Civil, Faculdade de Engenharia e Computação, Universidade de Itaúna

${ }^{3}$ Doutorando em Estatística e Experimentação Agropecuária, Departamento de Estatística, Universidade Federal de Lavras;

${ }^{4}$ Mestrando em Engenharia de Sistemas e Automação, Departamento de Engenharia de Sistemas e Automação,

Universidade Federal de Lavras; ${ }^{5}$ Doutorando em Estatística e Experimentação Agropecuária, Departamento de Estatística, Universidade Federal de Lavras; ${ }^{6}$ Graduanda em Engenharia Civil - Faculdade de Engenharia e Computação - Universidade

de Itaúna; ${ }^{7}$ Mestre em Engenharia de Sistemas e Automação, Departamento de Engenharia de Sistemas e Automação,

Universidade Federal de Lavras; ${ }^{8}$ Graduando em Engenharia Mecânica, Faculdade Pitágoras, Campus Divinópolis; 9,10, 11 \&

${ }^{12}$ Docente do curso de Engenharia Civil, Universidade Estadual de Minas Gerais

\section{ARTICLE INFO}

\section{Article History:}

Received $10^{\text {th }}$ April, 2021

Received in revised form

$19^{\text {th }}$ May, 2021

Accepted $02^{\text {nd }}$ June, 2021

Published online $28^{\text {th }}$ July, 2021

\section{Key Words:}

Geoestatística, Krigagem de Blocos, Médias Espaciais, Semivariogramas Médios.

*Corresponding author:

Ícaro Viterbre Debique Sousa

\begin{abstract}
RESUMO
Diversas áreas da ciência, tais como ambientais e biológicas, possuem dados com variações no espaço. Normalmente, mensuram-se somente as variações utilizando procedimentos estatísticos que dispensam as interações existentes entre as dimensões do espaço. Para se abordar a espacialidade tem-se a Geoestatística, que, com o uso do semivariograma,detecta a dependência espacial de um fenômeno. A Geoestatística utiliza-se de um preditor pontual. Neste trabalho é tratado um sistema de predição para toda uma região, denominado krigagem de blocos, que tem o intuito de predizer o valor médio do fenômeno no local de estudo. A principal complexidade desta técnica é a modelagem dos semivariogramas médios, pois atualmente essa necessidade é realizada somente de maneira numérica, sendo assim contendo erros de aproximação. Apresenta-se a construção dos semivariogramas médios analiticamente e com os resultados obtidos, é possível trabalhar em qualquer dimensão de região sem apresentar erros numéricos, com a total precisão de uma solução analítica que ainda não havia sido apresentada na literatura recorrente sobre krigagem de blocos. Toda a técnica para se construir os semivariogramas médios de maneira analítica é apresentada com o foco em modelar os semivariogramas médios para os modelos lineares.
\end{abstract}

Copyright C 2021, Ícaro Viterbre Debique Sousa et al. This is an open access article distributed under the Creative Commons Attribution License, which permits unrestricted use, distribution, and reproduction in any medium, provided the original work is properly cited.

Citation: Ícaro Viterbre Debique Sousa, Heron Viterbre Debique Sousa, Antonio Mendes Magalhães Júnior et al. "Construção Analítica de Semivariogramas Médios Lineares para Krigagem de Blocos”, International Journal of Development Research, 11, (07), 48917-48923.

\section{INTRODUÇÃO}

Existe uma grande necessidade de predizer determinados fenômenos, tais como relacionados à mineração, clima e ciências agrárias. Estes fenômenos muitas vezes são caracterizados por possuírem dependência espacial; sendo assim, necessitam de uma técnica estatística específica para sua modelagem. Um dos primeiros a observar de maneira profunda a necessidade de se explorar os dados com uma análise espacial foi Krige (1951). Enquanto trabalhava nas minas de ouro da África do Sul, percebeu que não havia o entendimento do fenômeno se não considerasse a distância entre as amostras, ou seja, se não considerasse a variação espacial. Posteriormente, os estudos de Matheron (1971) desenvolveram os conceitos básicos da Geoestatística, ferramenta utilizada para a predição de fenômenos contínuos no espaço por meio de um interpolador próprio denominado krigagem, nome dado em homenagem a Daniel Krige. O objetivo principal é apresentar a construção analítica dos semivariogramas 
médios para o modelo linear. Com a realização do propósito do trabalho, é possível construir semivariogramas médios para regiões retangulares para qualquer valor de lados, diferente dos principais autores que ao utilizar métodos numéricos limitaram-se a retângulos de lados prefixados. Também é possível definir o caminho de cálculo a ser trabalhado para qualquer modelo de semivariograma, visto que se apresentará o raciocínio, as equações e os métodos para tal cálculo. Adentrando no estudo da média espacial, a médiase trata da medida de posição mais comum, intensa e bastante utilizada. Valores médios podem ser importantes para o pesquisador ou mesmo a indústria. Suponha uma empresa de mineração que pretenda extrair algum material de uma determinada região. Em sua análise de dados, obter a informação do valor médio da quantidade de material a ser extraído pode ser importante em tomadas de decisão, em que os custos da extração devem ser menores do que as vendas do material, tornando a média uma informação importante nesse momento. Os conceitos comuns referentes a média não consideram a dependência espacial dos dados, mas é natural se imaginar que a dependência espacial deve ser levada em consideração no que se denomina médias espaciais.

Para a análise deste estudo, é importante compreender a ideia de média espacial em um sistema tridimensional, que pode ser representadapela função $f(x, y)$ em um domínio $D=\{(\mathrm{x}, \mathrm{y}) \in \mathbb{R} 2\}$ e, dada uma região $B$ abaixo da superfície da função $f(x, y)$ conforme a Figura 1 .

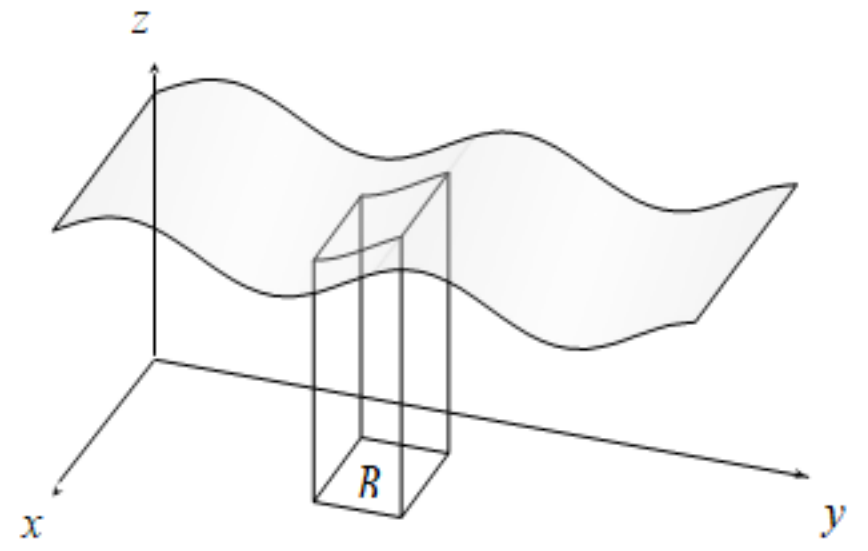

Fonte: o autor (2020)

Figura 1. Região $B$ abaixo da função $f(x, y)$

Para se calcular o volume do sólido Vzrepresentado na figura1, Stewart (2001) apresenta que pode ser obtido pela integral (1.1) abaixo dessa região.

$V z=\iint_{B} f(x, y) d x d y$

Na Figura 2 o sólido abaixo do gráfico da função possui variações em sua altura, ou seja, possui diversas alturas $h$ ao longo de toda área $B$; são infinitas alturas, isso impede que seja possível calcular o volume com conhecimentos de geometria comuns.

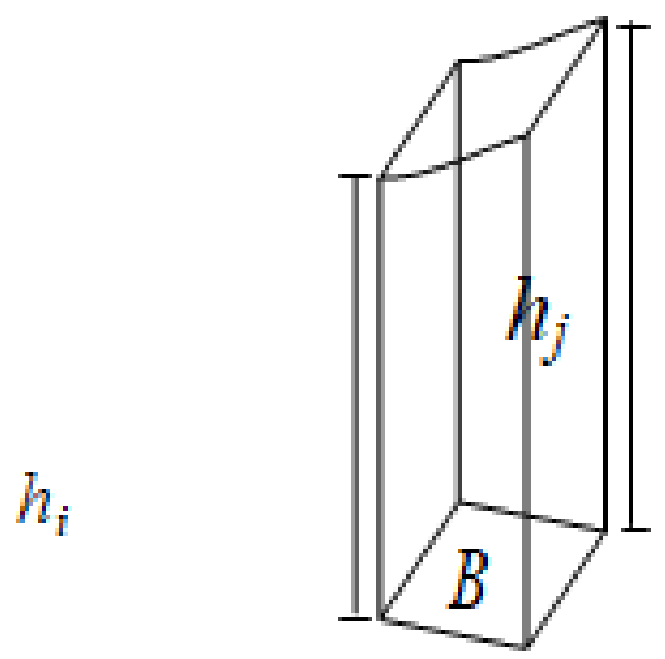

Fonte: o autor (2020)

Figura 2. Sólido com área da base $B$ e alturas $h$ 's

Se é possível obter a altura média $\bar{h}$, conforme apresentado na figura 3 podemos definir a equação do volume $\square \square(1.1)$ de uma nova maneira como apresentado na equação (1.2). 


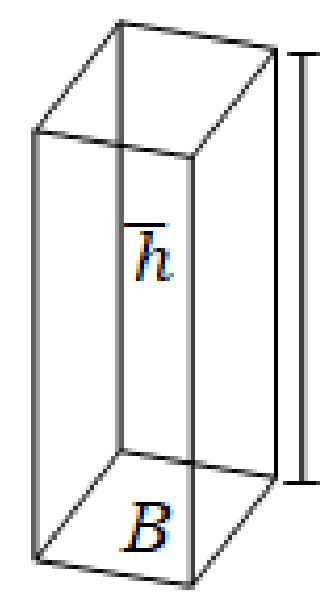

Fonte: o autor (2020)

\section{Figura 3. Sólido com área da base $B$ e altura $\bar{h}$}

Assim, em que $|B|$ é a área da região abaixo da superfície.

$V z=\iint_{B} f(x, y) d x d y=\bar{h}|B|$

Podemos definir a altura média $(\bar{h})$, ou seja, o valor médio da função $f(x, y)$, dado por:

$\bar{h}=\bar{f}(x, y)=\frac{V z}{|B|}=\frac{1}{|B|} \iint_{B} \quad f(x, y) d x d y$

Aequação (1.3) representa o valor médio de uma função $f$ de duas variáveis em um retângulo $B$ contido em seu domínio. Para a média espacial de uma variável aleatória, uma função em um espaço bidimensional ou tridimensional, os valores médios das mesmas estão relacionados a integral daquela função dividida pela região que ela ocorre. Essa ideia é construída para qualquer função e é naturalmente observável. No que corresponde a krigagem de blocos, ela surge na mineração, quando ao dividir uma região a ser minerada em diversos blocos, é necessário estimar a quantidade de material em cada bloco. Por esta demanda surge o nome krigagem de blocos; em planos de duas dimensões também pode ser chamada de krigagem de área. Esta demanda implica em calcular a média de variáveis aleatórias no espaço. Com a Geoestatística pode-se estimar a média do bloco de cubagem por meio da discretização do bloco em pontos, que podem ser avaliados individualmente e depois compostos para o bloco, ou então diretamente, calculando-se os vetores médios dos sistemas de equação. Essa alternativa da krigagem ordinária é denominada krigagem de bloco (Yamamoto e Landim, 2015). Considere o processo $\left\{Z(s): s \in A \subset \mathbb{R}^{2}\right\}$ que possui uma sub-região retangular $B$ de lados $P$ e $Q$, apresentado na figura4. Possuímos as amostras $\{\mathrm{Z}$ (s1), Z(s2), Z(s3), ...,Z(sn)\}e não estamos interessados em predizer um ponto $Z\left(s_{0}\right)$, mas sim um valor médio da região $B$, ou seja, o valor médio da variável aleatória na região $B, o$ valor $\bar{Z}(B)$.

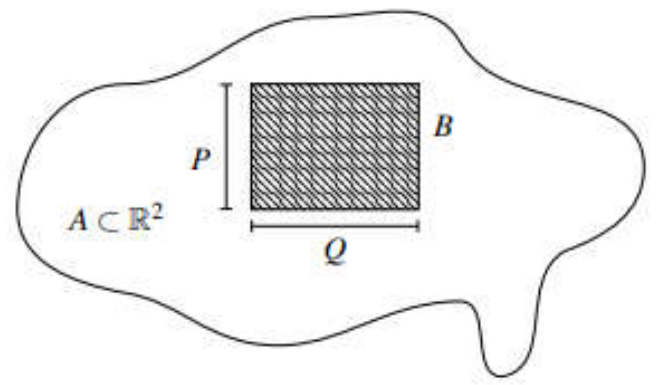

Fonte: o autor (2020)

Figura 4. Região $B$ de lados $P$ e $Q$

Schabenberger e Gotway (2017) definem $\bar{Z}(B)$ como:

$\bar{Z}(B)=\frac{1}{|B|} \int_{B} \quad Z(s) d s$

em que $|B|$ representa a área da região $B$. Ainda em Schabenberger e Gotway (2017), é apresentado o preditor para a krigagem de blocos, que é construído de forma análoga a krigagem pontual e é dado por:

$\bar{Z}(B)=\sum_{i=1}^{N} \quad \lambda_{i} Z\left(\boldsymbol{s}_{i}\right)$

Assim como na krigagem pontual, $N$ é o número de valores coletados $Z\left(\boldsymbol{s}_{\boldsymbol{i}}\right), \lambda_{i}$ são os pesos associados a cada valor. 
Na krigagem de blocos, os valores dos pesos $\lambda_{i}$ devem possuir $\sum_{i=1}^{N} \lambda_{i}=1$.Assim, como na krigagem ordinária, é necessário suprir a condição de não-tendenciosidade e variância mínima, que, sob a hipótese intrínseca, implica em minimizar $E[\bar{Z}(B)-$ $\left.\sum_{i=1}^{N} \lambda_{i} Z\left(\boldsymbol{s}_{\boldsymbol{i}}\right)\right]^{2}$,Burgess e Webster (1980a) apresentam a equação matricial para se obter os pesos $\lambda_{i}$ :

$\left[\begin{array}{ccccc}\gamma\left(\boldsymbol{s}_{1}, \boldsymbol{s}_{1}\right) & \gamma\left(\boldsymbol{s}_{1}, \boldsymbol{s}_{2}\right) & \cdots & \gamma\left(\boldsymbol{s}_{1}, \boldsymbol{s}_{\boldsymbol{n}}\right) & 1 \\ \gamma\left(\boldsymbol{s}_{2}, \boldsymbol{s}_{1}\right) & \gamma\left(\boldsymbol{s}_{2}, \boldsymbol{s}_{2}\right) & \cdots & \gamma\left(\boldsymbol{s}_{2}, \boldsymbol{s}_{\boldsymbol{n}}\right) & 1 \\ \vdots & \vdots & \ddots & \vdots & \vdots \\ \gamma\left(\boldsymbol{s}_{\boldsymbol{n}}, \boldsymbol{s}_{1}\right) & \gamma\left(\boldsymbol{s}_{\boldsymbol{n}}, \boldsymbol{s}_{2}\right) & \cdots & \gamma\left(\boldsymbol{s}_{\boldsymbol{n}}, \boldsymbol{s}_{\boldsymbol{n}}\right) & 1 \\ 1 & 1 & & 1 & 0\end{array}\right]-1\left[\begin{array}{c}\bar{\gamma}\left(\boldsymbol{s}_{1}, B\right) \\ \bar{\gamma}\left(\boldsymbol{s}_{2}, B\right) \\ \vdots \\ \bar{\gamma}\left(\boldsymbol{s}_{\boldsymbol{n}}, B\right) \\ 1\end{array}\right]=\left[\begin{array}{c}\lambda_{1} \\ \lambda_{2} \\ \vdots \\ \lambda_{n} \\ \emptyset\end{array}\right]$

Podemos simplificar a equação (2.3) na equação (2.4).

$A^{-1} c=\lambda$

Seria muito comum imaginar uma krigagem pontual e, posteriormente, utilizando os pontos krigados poderia obter a média desta variável. Esse pensamento possui fundamentos, mas perde em comparação com a krigagem de blocos, pois a krigagem de blocos utiliza-se de um estimador sem viés, enquanto a média pela krigagem pontual possui um erro, pois não seria possível utilizar os infinitos pontos daquela região, falha essa corrigida pela krigagem de blocos. Na equação matricial (2.4) o vetorc apresenta a semivariância entre os pontos $s_{1}, \ldots, s_{n} \mathrm{e}$ a região $B$ que se deseja estimar a média da variável aleatória, diferentemente da krigagem ordinária, onde na equação matricial o vetor $b$ apresenta a semivariância entre os pontos coletados e o ponto a ser predito. Para a krigagem de blocos, deve-se então estimar o semivariograma para os pontos em relação a região. Essa se apresenta como a maior dificuldade da krigagem de blocos. Faz-se então a necessidade da construção do semivariograma médio entre um ponto e todos os pontos dentro da área, chamado de semivariograma médio para o ponto $s_{i}$ e a região $B$. A construção do semivariograma médio é a maior complexidade e diferencial da krigagem de blocos em relação a qualquer outro tipo de krigagem. Se associarmos ao semivariograma construído na krigagem ordinária, na qual existia a associação de ponto a ponto, considerava-se somente a distância euclidiana entre eles. Agora, o semivariograma é entre um ponto e uma área e não existe maneira de se utilizar somente uma distância. A semivariância entre um ponto e uma área é apresentada de maneira numérica por Clark (1979), mas em Oliveira (1991) apresenta-se as definições do semivariograma médio e a maneira para se obter os resultados analiticamente, onde se aplica esta definição geral apenas para o modelo linear. Este trabalho tem como principal objetivo demonstrar a construção do semivariograma médio para outros modelos de semivariograma e serão apresentados na metodologia.

\section{MATERIAIS E MÉTODOS}

Ao se realizar a krigagem de blocos, é necessária a construção de um semivariograma médio da região. Essa etapa é o que difere da krigagem ordinária e, assim, é a mais importante para a krigagem de blocos. Clark (1979) e Journel e Huijbregts (1978) apresentam apenas resultados numéricos para o semivariograma médio, mas nesta dissertação apresenta-se agora o método qual para a construção analítica dos mesmos, baseado em Oliveira (1991). Seja um processo estocástico que satisfaz a hipótese intrínseca, com semivariograma isotrópico e efeito pepita $C_{0}$. Se considerarse uma subregião $B$ retangular de lados $P$ e $Q$, então o semivariograma médio entre o ponto $s$ e a região $B$ é descrita por Oliveira (1991) é dado na equação (2.5).

$\bar{\gamma}(s, B)=\frac{1}{P Q} \int_{0}^{\sqrt{P^{2}+Q^{2}}} M(h) \gamma(h) d h$

em que $P$ e $Q$ são os lados do retângulo que se deseja obter a semivariância média, $\gamma(h)$ o semivariograma estimado para os pontos amostrados e $M(h)$ é o comprimento do arco formado pelo círculo no entorno do ponto sendo que esteja contido somente dentro da região de estudo. É possível a melhor visualização de $M(h)$ com a figura 5.

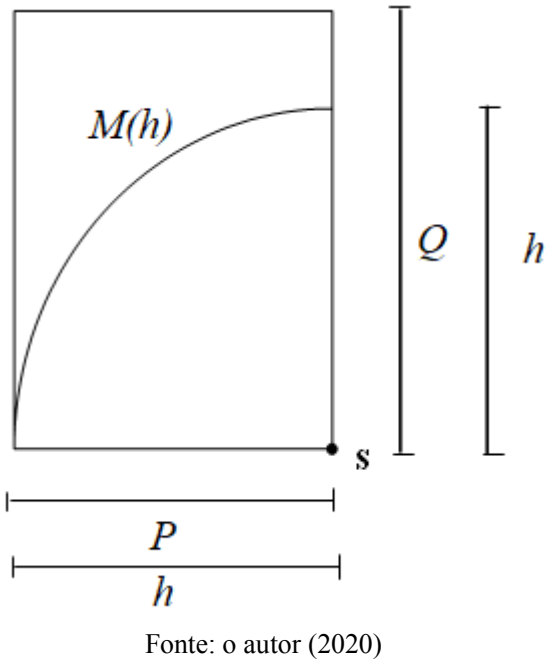

Figura 5. Semivariograma médio entre um ponto e uma área 
No segundo estágio, se $P<h \leq Q$, o valor de $M(h)$ extrapola as dimensões do retângulo de lados $P$ e $Q$, mas somente em sua base $P$, gerando assim a parte da curva denominada $M(h)^{*} \mathrm{e}$, assim, $M(h)=\frac{\pi h}{2}-\arccos \quad\left(\begin{array}{l}P \\ h\end{array}\right) h$, conforme apresentado na figura 7 .

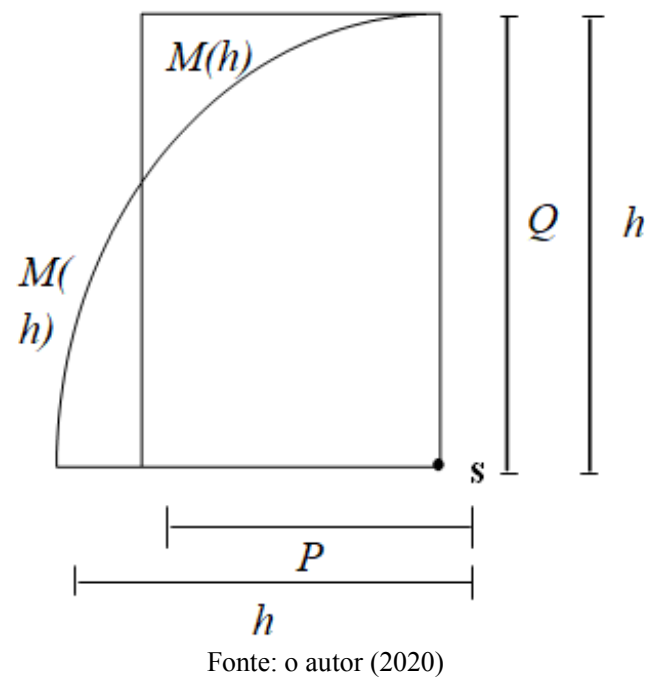

Figura 7. Curva $\boldsymbol{M}(\boldsymbol{h})$ para o intervalo $\boldsymbol{P}<h \leq Q$

No terceiro estágio, quando $Q<h \leq \sqrt{P^{2}+Q^{2}}$, o valor de $M(h)$ extrapola as dimensões do retângulo de lados $P$ e $Q$ em ambas direções, gerando assim as partes da curva $M(h) *$ e $M(h) * *$. Então $M(h)=\frac{\pi h}{2}-\arccos \quad\left(\begin{array}{l}P \\ h\end{array}\right) h-\arccos \left(\begin{array}{l}Q \\ h\end{array}\right) h$, conforme apresentado na figura 8 .

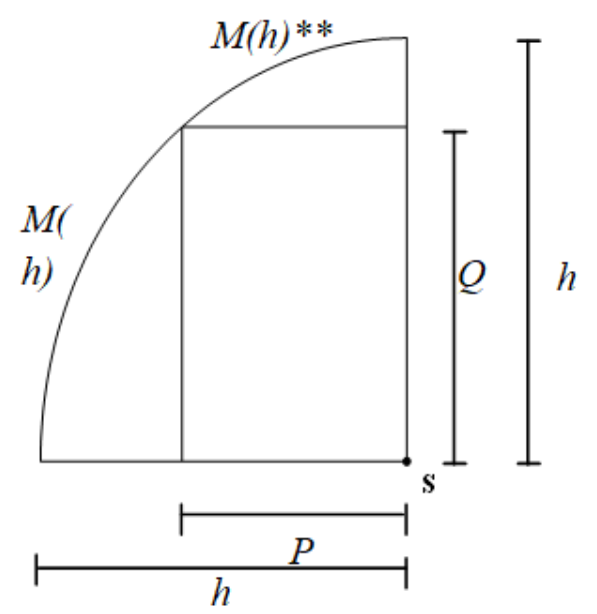

Figura 8. Curva $M(h)$ para o intervalo $Q<h \leq \sqrt{P^{2}+Q^{2}}$

Com os estudos de Oliveira (1991) podemos notar que a construção da curva $M(h)$ acontece somente dentro do retângulo $P$ e $Q$ e em alguns estágios essa curva pode extrapolar essas dimensões, gerando assim o que foi denominado de $M(h)^{*}$ e $M(h)^{* *}$. Podemos notar nas figuras 6,7 e 8 que $M(h)=\frac{\pi h}{2}, M(h)^{*}=\arccos \quad\left(\begin{array}{l}P \\ h\end{array}\right)$ h e $M(h)^{* *}=\arccos \left(\begin{array}{l}Q \\ h\end{array}\right) h$.

Logo, a equação (2.5) pode ser desmembrada na soma das equações (4.6), (4.7) e (4.8).

$\int_{0}^{p} \frac{\pi h}{2} \gamma(h) d h$

$\int_{P}^{Q}\left[\frac{\pi h}{2}-\arccos \left(\begin{array}{l}P \\ h\end{array}\right) h\right] \gamma(h) d h$

$\int_{Q}^{\sqrt{P^{2}+Q^{2}}}\left[\frac{\pi h}{2}-\arccos \left(\begin{array}{l}P \\ h\end{array}\right) t h-\arccos \left(\begin{array}{l}Q \\ h\end{array}\right) h\right] \gamma(h) d h$

Assim, toda a construção do semivariograma médio para krigagem de blocos apresentada por Oliveira (1991) pode ser resumida na equação (2.9), ou seja,

$\bar{\gamma}(s, B)=\frac{1}{P Q} \int_{0}^{P} \frac{\pi h}{2} \gamma(h) d h+$ 
$+\frac{1}{P Q} \int_{P}^{Q}\left[\frac{\pi h}{2}-\arccos \left(\begin{array}{l}P \\ h\end{array}\right) h\right] \gamma(h) d h+$

$+\frac{1}{P Q} \int_{Q}^{\sqrt{P^{2}+Q^{2}}}\left[\frac{\pi h}{2}-\arccos \left(\begin{array}{l}P \\ h\end{array}\right) h-\arccos \left(\begin{array}{l}Q \\ h\end{array}\right) h\right] \gamma(h) d h$

Dessa maneira, é possível obter a semivariância média entre um ponto situado no vértice do bloco e o bloco retangular de lados $P$ e $Q$. A mesma ideia pode ser abordada para outras geometrias. Neste trabalho todos os blocos de krigagem são retangulares. Todo fenômeno possui um semivariograma que mapeia a dependência espacial. Com essa informação é possível, com a equação (2.9), obter o semivariograma médio para qualquer região de lados $P$ e $Q$ do fenômeno.

Recursos computacionais: O software R Core Team (2019) foi utilizado para a obtenção dos resultados e comparação com os obtidos na literatura. Tem-se como principal intuito utilizar o R, pois se trata de uma plataforma livre, e é de fácil acesso a outros pesquisadores.

\section{RESULTADOS E DISCUSSÃO}

Sendo o objetivo deste trabalho enunciar os conceitos básicos da krigagem de blocos e com as informações apresentadas por Oliveira (1991), a construção dos semivariogramas médios entre pontos e regiões retangulares, tem-se então como resultado as equações para os modelos lineares. O modelo linear foi escolhido pois Journel e Huijbregts (1978) expõemo seu resultado. Dada que a função do variograma $\hat{y}(\square)$ a ser estudada é a linear, definida por:

$\hat{\mathrm{y}}(h)=\left\{\begin{array}{c}0, \text { para } h=0 \\ C_{0}+\text { ah, para } h>0\end{array}\right.$

Utilizando a equação (2.5) e seu desmembramento, podemos obter o semivariograma médio entre um ponto $s$ no vértice da região $B$ e a região $B$ para o modelo linear (3.1); temos a seguinte equação:

$\bar{\gamma}(\boldsymbol{s}, B)_{L i n}=C_{0}+\frac{1}{P Q} \int_{0}^{\sqrt{P^{2}+Q^{2}}} M(h) a h d h$

Em todo este trabalho é utilizado a nomenclatura $\bar{\gamma}(s, B)_{\text {Lin }}$ para o semivariograma médio do modelo linear. Desmembrando cada item temos então as seguintes integrais conforme apresentado na equação (2.9) para solucionar e obter o semivariograma médio para o modelo linear.

$\frac{1}{P Q} \int_{Q}^{P} \frac{\pi h}{2} a h d h$

$\frac{1}{P Q} \int_{P}^{Q}\left[\frac{\pi h}{2}-\arccos \left(\begin{array}{l}P \\ Q\end{array}\right) h\right] \operatorname{ahdh}$

$\frac{1}{P Q} \int_{Q}^{\sqrt{P^{2}+Q^{2}}}\left[\frac{\pi h}{2}-\arccos \left(\begin{array}{l}P \\ Q\end{array}\right) h-\arccos \left(\begin{array}{l}Q \\ h\end{array}\right) h\right] \operatorname{ahdh}$

Seguindo o objetivo que é apresentar as equações de semivariogramas médios para krigagem de blocos, é possível apresentar como resultado a equação do semivariograma médio para um modelo linear. Propomos que o semivariograma médio linear é dado pela equação (3.6):

$\bar{\gamma}(\boldsymbol{s}, B)_{\operatorname{Lin}}=C_{0}+\frac{a}{6}\left(2 \sqrt{P^{2}+Q^{2}}+\frac{P^{2}}{Q} \ln \left|\frac{Q+\sqrt{P^{2}+Q^{2}}}{P}\right|+\frac{Q^{2}}{P} \ln \left|\frac{P+\sqrt{P^{2}+Q^{2}}}{Q}\right|\right)$

O resultado obtido pode ser atestado em Journel e Huijbregts (1978), denominando a função como $H(L, l)$ e em Oliveira (1991) denominando a função como $\bar{\gamma}(s, r)$. Os dois autores não apresentam a demonstração analítica da obtençãoda função.

\section{CONCLUSÃO}

Por este trabalho é possível compreender a construção da semivariância média entre um ponto e uma região, assim, pode-se construir a semivariância média para qualquer modelo em qualquer região. Neste foi apresentado essa construção para regiões retangulares. Mesmo em face da atual possibilidade da solução numérica das integrais, visto a facilidade do uso computacional, as expressões analíticas (algébricas) continuam tendo seu valor, pois é uma solução exata, geral e independente de métodos numéricos adotados. A precisão das resoluções analíticas dáuma grande confiança no trabalho desenvolvido. É possível concluir que a construção do modelo de semivariograma médio linear é precisa e se iguala ao apresentado na literatura existente. Construir o semivariograma médio linear se caracteriza um avanço nos métodos da krigagem de blocos.

\section{REFERÊNCIAS}

Burgess, T., Webster, R. 1980. “Optimal interpolation and isarithmic mapping of soil properties: Ii block kriging”. Journal of Soil Science, Wiley Online Library, v. 31, pp. 333-341.

Clark, I. 1979. Practical Geostatistics. (S.1.): Applied Science Publishers London, 1979. v. 3.

Journel, A. G., Huijbregts, C. J.1978. Mining geostatistics. (S.1.): Academic press London, 1978. v. 600. 
Krige, D. G. 1951. "A statistical approach to some basic mine valuation problems on the witwatersrand". Journal of the Southern African Institute of Mining and Metallurgy,v. 52, pp. 119-139.

Matheron, G. 1971. "The theory of regionalised variables and its applications". Les Cahiers du Centre de Morphologie Mathématique, v. 5, p. 212.

Oliveira, M. S. d. 1991. Planos amostrais para variáveis espaciais utilizando geoestatística. Dissertação de Mestrado, Universidade Estadual de Campinas, 1991.

Schabenberger, O., Gotway, C. A. 2017. Statistical methods for spatial data analysis. (S.1.): CRC press, 2017.

Yamamoto, J. K., Landim, P. M. B. 2015. Geoestatística: conceitos e aplicações. (S.1.): Oficina de textos, 2015. 